\title{
Path integral methods for reaction rates in complex systems
}

\author{
Joseph E. Lawrence and David E. Manolopoulos \\ Department of Chemistry, University of Oxford, \\ Physical and Theoretical Chemistry Laboratory, \\ South Parks Road, Oxford OX1 3QZ, UK
}

\begin{abstract}
We shall use this introduction to the Faraday Discussion on quantum effects in complex systems to review the recent progress that has been made in using imaginary time path integral methods to calculate chemical reaction rates. As a result of this progress, it is now routinely possible to calculate accurate rate constants including quantum mechanical zero point energy and tunnelling effects for arbitrarily complex (anharmonic and multi-dimensional) systems. This is true in the adiabatic (Born-Oppenheimer) limit, in the non-adiabatic (Fermi Golden Rule) limit, and everywhere between these two limits in the normal Marcus regime. Quantum mechanical effects on reaction rates can be enormous, even at room temperature, and the problem of including these effects in simulations of a wide variety of chemical reactions in complex systems has now effectively been solved.
\end{abstract}




\section{INTRODUCTION}

There can hardly be a situation in which quantum mechanical zero point energy and tunnelling effects are more significant than they are in chemical reaction rates. Every undergraduate chemist knows that changes in zero point energy between the reactants and the transition state give rise to kinetic isotope effects in chemical reactions, and that tunnelling through the reaction barrier can increase the rate of a proton (or hydrogen atom, or hydride) transfer reaction at room temperature by several orders of magnitude. 1 These primary quantum mechanical effects are ubiquitous in reaction rates, and they are just as important in the condensed phase as they are in the gas phase. (There are also some more subtle quantum mechanical effects in chemical reactions, such as quantum reactive scattering resonances. ${ }^{2}$ However, these tend to be washed out in the condensed phase, and so are less relevant to the present Discussion about quantum effects in complex systems. In fact, they are pretty difficult to detect even in the gas phase, where they have only been detected so far in observables that are significantly more detailed than thermal rates. ${ }^{213}$ )

For gas phase reactions involving relatively few atoms, it is now possible to solve the Schrödinger equation exactly subject to scattering boundary conditions to obtain rate coefficients which automatically include all quantum mechanical effects. However, it is not possible to do this for condensed phase reactions and likely never will be, because of the exponential scaling of quantum mechanics with dimensionality. The only case we are aware of in which this exponential scaling can be avoided is in the calculation of the thermal equilibrium properties of systems composed of distinguishable particles. In this case one can exploit a well-known isomorphism ${ }^{4}$ between the quantum mechanical partition function and that of a classical ring polymer to obtain a computational scheme that scales only linearly with dimensionality.

The classical isomorphism forms the basis of the path integral molecular dynamics (PIMD) method,,$\sqrt[5]{ }$ which has been used for over thirty years to include nuclear quantum effects in simulations of the static equilibrium properties of condensed phase systems. More recently, it has become apparent that the same isomorphism can be used to approximate the dynamical properties of these systems, such as diffusion coefficients and chemical reaction

rate coefficients. In particular, ring polymer molecular dynamics ${ }^{6}$ (RPMD) reaction rate theory ${ }^{788}$ provides a convenient way to estimate the rates of electronically adiabatic reac- 
tions in condensed phase systems, with the inclusion of zero point energy and tunnelling effects. For electronically non-adiabatic reactions, such as electron transfer reactions in the Fermi Golden Rule limit, one can use a much older imaginary time path integral theory due to Wolynes. $\stackrel{9}{9}$ And between the adiabatic and non-adiabatic limits, one can use a simple interpolation formula to estimate the rate from the results of separate RPMD rate theory and Wolynes theory calculations. 10

In the following sections of this review, we shall summarise each of these developments in turn, with an emphasis on explaining why it is that imaginary time path integral methods are so effective for calculating quantum reaction rates. To keep the presentation as simple as possible, we shall only give the equations for one-dimensional models of chemical reactions. One of the greatest strengths of the imaginary time path integral formulation is that it is straightforward to write down the equations that apply to more complicated multi-dimensional problems, simply by adding an extra index to each bead of the ring polymer necklace that runs over the Cartesian coordinates of each atom. ${ }^{11}$ We shall also try to illustrate the physical effects that imaginary time path integrals are capable of capturing with appropriate diagrams as we present the material, so as to make the presentation as accessible as possible.

\section{THE CLASSICAL ISOMORPHISM}

Consider a one-dimensional system with the Hamiltonian

$$
\hat{H}=\hat{T}+\hat{V}=\frac{\hat{p}^{2}}{2 m}+V(\hat{q})
$$

The canonical quantum mechanical partition function is $Q(T)=\operatorname{tr}\left[e^{-\beta \hat{H}}\right]$, where $\beta=$ $1 / k_{\mathrm{B}} T$. The remarkable fact is that this can be written exactly as a classical partition function of a ring polymer comprising $n \rightarrow \infty$ copies of the physical system coupled by harmonic springs. $\frac{4}{}$ The result is simply

$$
Q(T)=\lim _{n \rightarrow \infty} Q_{n}(T)
$$

where

$$
Q_{n}(T)=\frac{1}{(2 \pi \hbar)^{n}} \int \mathrm{d}^{n} \mathbf{p} \int \mathrm{d}^{n} \mathbf{q} e^{-\beta_{n} H_{n}(\mathbf{p}, \mathbf{q})}
$$


and

$$
H_{n}(\mathbf{p}, \mathbf{q})=\sum_{j=1}^{n}\left[\frac{p_{j}^{2}}{2 m}+\frac{1}{2} m \omega_{n}^{2}\left(q_{j}-q_{j+1}\right)^{2}+V\left(q_{j}\right)\right],
$$

with $\beta_{n}=\beta / n, \omega_{n}=1 / \beta_{n} \hbar$, and $q_{n+1} \equiv q_{1}$. The proof is straightforward, using

$$
\begin{aligned}
\operatorname{tr}\left[e^{-\beta \hat{H}}\right] & =\operatorname{tr}\left[\left(e^{-\beta_{n} \hat{H}}\right)^{n}\right] \\
& =\int_{-\infty}^{\infty} \mathrm{d} q_{1} \cdots \int_{-\infty}^{\infty} \mathrm{d} q_{n}\left\langle q_{1}\left|e^{-\beta_{n} \hat{H}}\right| q_{2}\right\rangle \cdots\left\langle q_{n}\left|e^{-\beta_{n} \hat{H}}\right| q_{1}\right\rangle,
\end{aligned}
$$

and

$$
\begin{aligned}
\left\langle q_{j}\left|e^{-\beta_{n} \hat{H}}\right| q_{j+1}\right\rangle & \simeq\left\langle q_{j}\left|e^{-\beta_{n} \hat{V} / 2} e^{-\beta_{n} \hat{T}} e^{-\beta_{n} \hat{V} / 2}\right| q_{j+1}\right\rangle \\
& =\frac{1}{(2 \pi \hbar)} \int_{-\infty}^{\infty} \mathrm{d} p_{j} e^{-\beta_{n}\left[p_{j}^{2} / m+m \omega_{n}^{2}\left(q_{j}-q_{j+1}\right)^{2}+V\left(q_{j}\right)+V\left(q_{j+1}\right)\right] / 2} .
\end{aligned}
$$

The approximation in Eq. (6) has an error of $O\left(\beta_{n}^{3}\right)=O\left(1 / n^{3}\right)$. Since this approximation is made $n$ times when Eqs. (5) and (6) are combined to give Eq. (3), the overall error in $Q_{n}(T)$ is $O\left(1 / n^{2}\right)$. This error tends to zero as $n \rightarrow \infty$, and it becomes negligible for sufficiently large $n$. (In practice, imaginary time path integral simulations of systems containing hydrogen atoms are typically well converged with $n \approx 50$ ring polymer beads at room temperature, and with twice as many beads at half the temperature.)

It is important to stress that the classical isomorphism comes with a physical picture: particles "swell" as a result of thermal quantum fluctuations as illustrated in Fig. 1. For a free particle moving in one dimension with $V(q)=0$, it is straightforward to work out the thermally averaged radius of gyration of the ring polymer

$$
\Delta q=\left\langle\frac{1}{n} \sum_{j=1}^{n}\left(q_{j}-\bar{q}\right)^{2}\right\rangle^{1 / 2}
$$

around its centroid

$$
\bar{q}=\frac{1}{n} \sum_{j=1}^{n} q_{j}
$$

where the thermal average is defined as

$$
\langle\cdots\rangle=\frac{1}{(2 \pi \hbar)^{n} Q_{n}(T)} \int \mathrm{d}^{n} \mathbf{p} \int \mathrm{d}^{n} \mathbf{q} e^{-\beta_{n} H_{n}(\mathbf{p}, \mathbf{q})}(\cdots) .
$$

This turns out to be

$$
\Delta q=\sqrt{\frac{\beta \hbar^{2}}{12 m}\left(1-\frac{1}{n^{2}}\right)} \sim \frac{\hbar}{\sqrt{12 m k_{\mathrm{B}} T}} \text { as } n \rightarrow \infty .
$$




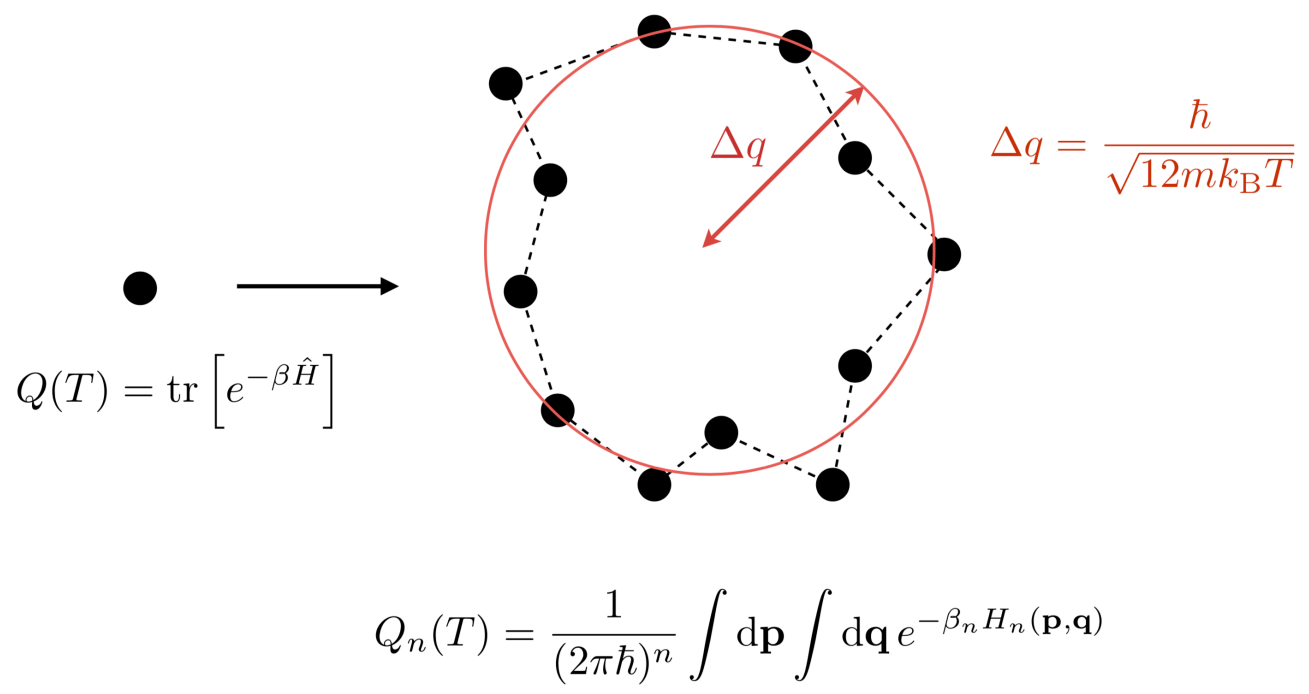

FIG. 1. The classical isomorphism

Hence the swelling is most pronounced for light atoms at low temperatures, and it shrinks to zero for heavy atoms at high temperatures (i.e., in the classical limit in which a single ring polymer bead suffices and Eq. (3) reduces to a purely classical partition function).

The physical significance of the ring polymer swelling was first highlighted by Chandler and Wolynes in their pioneering paper on the classical isomorphism, in which they attributed it to quantum dispersion arising from the uncertainty principle. ${ }^{[4}$ It has since been used to explain the origin of the quantum mechanical effects in a variety of condensed phase situations, including simulations of liquid para-hydrogen ${ }^{12}$ and the diffusion of hydrogen, deuterium and muonium atoms in water and ice, $\frac{13}{13}$ and to account for the competing quantum effects that arise in a wide variety of systems ranging from liquid water ${ }^{14}$ to clathrate hydrates. $\frac{15}{15}$

The swelling also provides a clear picture of how the classical isomorphism includes zero point energy and tunnelling effects, as illustrated in Fig. 2. Consider first the passage of a ring polymer over a potential energy barrier as shown in Fig. 2a. The classical probability that a thermal fluctuation will bring a particle to the top of the barrier is $\exp \left[-\beta V\left(q^{\ddagger}\right)\right]$, where $V\left(q^{\ddagger}\right)$ is the potential energy at the barrier top $\left(q=q^{\ddagger}\right)$. However, when the centroid of the ring polymer is at the top of the barrier, its beads are dispersed around $q^{\ddagger}$ by the 


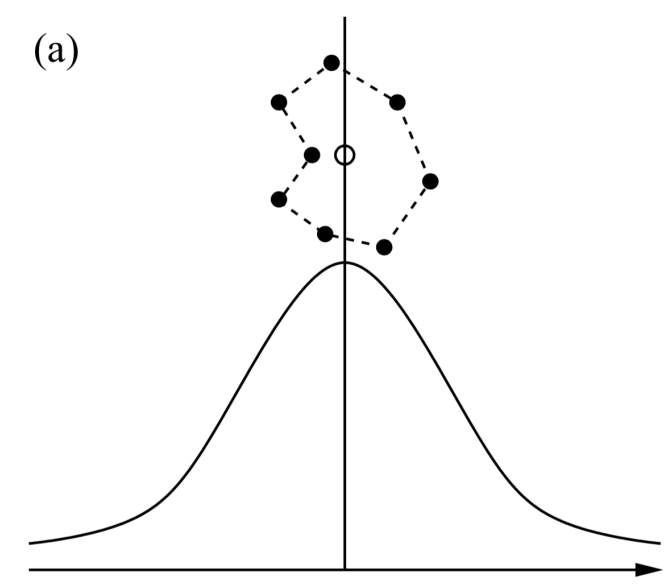

(b)

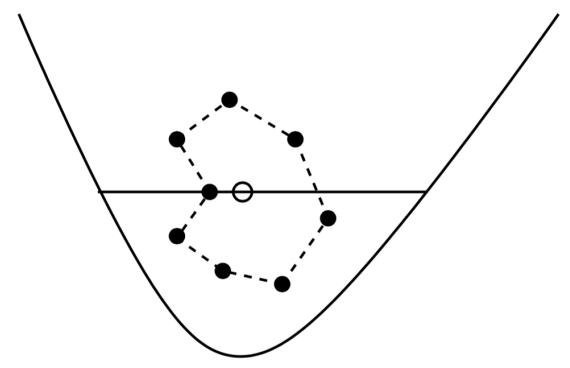

FIG. 2. Illustration of how the classical isomorphism captures (a) tunnelling and (b) zero point energy. See the text for a discussion of each panel.

swelling, and so each experience a lower potential energy. The average potential energy of the beads is therefore lower than that of the barrier top, and thus the probability that a thermal fluctuation will bring the centroid of the ring polymer to the top of the barrier, $\exp \left[-\beta_{n} \sum_{j=1}^{n} V\left(q_{j}\right)\right]$, is higher than in the classical case (recall that $\beta_{n}=\beta / n$ ). In other words, the ring polymer can pass through the barrier without the average potential energy of its beads reaching that of the barrier top, which provides a qualitative explanation of how the classical isomorphism captures tunnelling.

Now consider a ring polymer in a potential energy well as illustrated in Fig. 2b. Classically, a particle can be stationary at the bottom of the well, with no zero point energy. But when the centroid of the ring polymer is in this position, its beads are dispersed around the bottom of the well by the swelling, and so each experience a higher potential energy. The average potential energy of the beads is therefore now higher than that of the well minimum, and in the low-temperature limit (where the swelling is most pronounced) the thermal average of the increase gives the potential energy contribution to the zero point energy. (In fact, it should be obvious that the classical isomorphism is able to capture zero 
point energy, because it is consistent with the quantum mechanical partition function. In the low temperature limit, this reduces to $Q(T)=e^{-\beta E_{0}}$, which only depends on the zero point energy level $E_{0}$.)

These arguments have inevitably been somewhat qualitative. Among other things, we have based them on the swelling of the ring polymer in the free particle limit [Eq. (10)]. In reality, the swelling will be more pronounced when a ring polymer passes over a potential energy barrier (as in Fig. 2a), and less pronounced when it is confined in a potential energy well (as in Fig. 2b). However, both arguments are physically reasonable, and they do suffice to explain at least qualitatively how it is that the classical isomorphism captures tunnelling and zero point energy effects. The fact that these are the primary quantum mechanical effects in chemical reaction rates makes the reaction rate problem a "killer application" for the classical isomorphism, as we shall describe in the remaining sections of this review.

\section{RING POLYMER RATE THEORY}

Now consider a one-dimensional barrier transmission problem with a Hamiltonian of the form in Eq. (1), in which $V(q)$ tends to zero as $q \rightarrow-\infty$ (the reactant asymptote) and to a constant as $q \rightarrow \infty$ (the product asymptote). This provides the simplest possible model for a bimolecular chemical reaction. The exact quantum mechanical thermal rate coefficient for this reaction can be written as $\frac{16}{16}$

$$
k(T)=\frac{1}{Q_{r}(T)} \lim _{t \rightarrow \infty} c_{f s}(t)
$$

where $Q_{r}(T)=\sqrt{2 \pi m k_{\mathrm{B}} T} / h$ is the reactant partition function per unit length and

$$
c_{f s}(t)=\operatorname{tr}\left[e^{-\beta \hat{H} / 2} \hat{F} e^{-\beta \hat{H} / 2} e^{+i \hat{H} t / \hbar} \hat{h} e^{-i \hat{H} t / \hbar}\right]
$$

is a flux-side correlation function. Here the flux operator is defined as

$$
\hat{F}=\frac{i}{\hbar}[\hat{H}, \hat{h}]
$$

and the side operator as

$$
\hat{h}=h\left(\hat{q}-q^{\ddagger}\right),
$$

where $h(x)$ is a Heaviside step function. The side operator projects onto states on the product side of a configuration-space dividing surface between reactants and products (at 
$\left.q=q^{\ddagger}\right)$, and the flux operator is the Heisenberg time derivative of $\hat{h}$, which gives the flux onto the product states. The correlation function $c_{f s}(t)$ in Eq. (12) correlates the thermal flux through the dividing surface at time $0, e^{-\beta \hat{H} / 2} \hat{F} e^{-\beta \hat{H} / 2}$, with the projection onto the product side of the dividing surface at time $t, e^{+i \hat{H} t / \hbar} \hat{h} e^{-i \hat{H} t / \hbar}$.

It should be stressed that $k(T)$ in Eq. (11) is not a transition state theory approximation to the rate but rather the exact quantum mechanical rate constant. Any recrossing of the dividing surface at $q=q^{\ddagger}$ is automatically accounted for by taking the infinite time limit of $c_{f s}(t)$. It should also be stressed that, because of this, $k(T)$ is independent of the choice of the dividing surface (the choice of $q^{\ddagger}$ ). This is also true for the generalisation of Eq. (11) to multi-dimensional reactions, as can be shown using the quantum mechanical continuity equation (the fact that the stationary state flux through a closed surface is zero in any number of dimensions).

The classical limit of the rate constant has exactly the same form as Eq. (11),

$$
k^{\mathrm{cl}}(T)=\frac{1}{Q_{r}(T)} \lim _{t \rightarrow \infty} c_{f s}^{\mathrm{cl}}(t),
$$

in which $c_{f s}^{\mathrm{cl}}(t)$ is now a classical flux-side correlation function

$$
c_{f s}^{\mathrm{cl}}(t)=\frac{1}{2 \pi \hbar} \int \mathrm{d} p_{0} \int \mathrm{d} q_{0} e^{-\beta H\left(p_{0}, q_{0}\right)} \delta\left(q_{0}-q^{\ddagger}\right) \frac{p_{0}}{m} h\left(q_{t}-q^{\ddagger}\right) .
$$

Here $H(p, q)=p^{2} / 2 m+V(q)$ is the classical Hamiltonian and $q_{t} \equiv q_{t}\left(p_{0}, q_{0}\right)$ is the coordinate at time $t$ of a classical trajectory generated by this Hamiltonian that starts out at the initial phase space point $\left(p_{0}, q_{0}\right)$ at time 0 . The trace in Eq. (12) has become a phase space average, the quantum Boltzmann operator a classical Boltzmann factor, the quantum flux operator the classical flux through the dividing surface, $\mathrm{d} h\left(q_{0}-q^{\ddagger}\right) / \mathrm{d} t=\delta\left(q_{0}-q^{\ddagger}\right) p_{0} / m$, and the quantum side operator a simple step function $h\left(q_{t}-q^{\ddagger}\right)$ that counts trajectories on the product side of the dividing surface at time $t$.

As in the quantum mechanical case, the long-time limit in Eq. (15) allows for recrossing of the dividing surface and gives a rate constant that is rigorously independent of the choice of $q^{\ddagger}$. However, this rate constant does not include the effect of tunnelling through the reaction barrier, and its multi-dimensional generalisation does not allow for changes in zero point energy in modes orthogonal to the reaction coordinate as the reaction proceeds. So let us now return to the classical isomorphism, which fixes both of these problems. 
The RPMD rate coefficient for the same one-dimensional barrier transmission problem can be written in a variety of different ways, $\frac{78}{17}$ the most general of which is

$$
k^{\mathrm{RPMD}}(T)=\frac{1}{Q_{r}(T)} \lim _{t \rightarrow \infty} c_{f s}^{\mathrm{RPMD}}(t),
$$

where

$$
c_{f s}^{\mathrm{RPMD}}(t)=\frac{1}{(2 \pi \hbar)^{n}} \int \mathrm{d} \mathbf{p}_{0} \int \mathrm{d} \mathbf{q}_{0} e^{-\beta_{n} H_{n}\left(\mathbf{p}_{0}, \mathbf{q}_{0}\right)} \dot{h}\left[s\left(\mathbf{q}_{0}\right)\right] h\left[s\left(\mathbf{q}_{t}\right)\right],
$$

with

$$
\dot{h}[s(\mathbf{q})]=\delta[s(\mathbf{q})] \sum_{j=1}^{n} \frac{\partial s(\mathbf{q})}{\partial q_{j}} \cdot \frac{p_{j}}{m} .
$$

Here $s(\mathbf{q})=0$ is a dividing surface in the ring polymer configuration space between reactants and products, and $\mathbf{q}_{t} \equiv \mathbf{q}_{t}\left(\mathbf{p}_{0}, \mathbf{q}_{0}\right)$ is the configuration at time $t$ of a trajectory generated by the classical ring polymer Hamiltonian $H_{n}(\mathbf{p}, \mathbf{q})$ from the initial ring polymer phase space point $\left(\mathbf{p}_{0}, \mathbf{q}_{0}\right)$. As in the quantum mechanical and purely classical cases, the long time limit in Eq. (17) allows for recrossing of the dividing surface, and the rate coefficient $k^{\operatorname{RPMD}}(T)$ can be shown to be independent of how this dividing surface is defined, provided it separates reactants from products. (This actually follows quite straightforwardly from the fact that Eq. (17) is a purely classical rate coefficient in the extended ring polymer phase space.) However, unlike the classical case, Eq. (17) does allow for tunnelling along the reaction coordinate, and its multi-dimensional generalisation does allow for changes in zero point energy as the reaction proceeds, for the reasons we have discussed in Sec. II.

One of the simplest choices of the ring polymer dividing surface, which also happens to be the most appropriate in the high temperature limit, is $s(\mathbf{q})=\bar{q}-q^{\ddagger}$, where $\bar{q}$ is the centroid of the ring polymer defined in Eq. (8). With this choice, Eq. (18) becomes

$$
c_{f s}^{\mathrm{RPMD}}(t)=\frac{1}{(2 \pi \hbar)^{n}} \int \mathrm{d} \mathbf{p}_{0} \int \mathrm{d} \mathbf{q}_{0} e^{-\beta_{n} H_{n}\left(\mathbf{p}_{0}, \mathbf{q}_{0}\right)} \delta\left(\bar{q}_{0}-q^{\ddagger}\right) \frac{\bar{p}_{0}}{m} h\left(\bar{q}_{t}-q^{\ddagger}\right) .
$$

Here the (thermal) flux of ring polymer centroids through the dividing surface at time $t=0$ is correlated with the population of ring polymer centroids on the product side of the dividing surface at time $t$, as illustrated schematically in Fig. 3. Since this $c_{f s}^{\mathrm{RPMD}}(t)$ reduces to the classical flux-side correlation function in Eq. (16) when it is evaluated with just a single ring polymer bead $(n=1)$, it is clear that RPMD rate theory is pinned to classical rate theory in the high temperature limit, where one bead does indeed suffice and the classical rate coefficient becomes exact. 


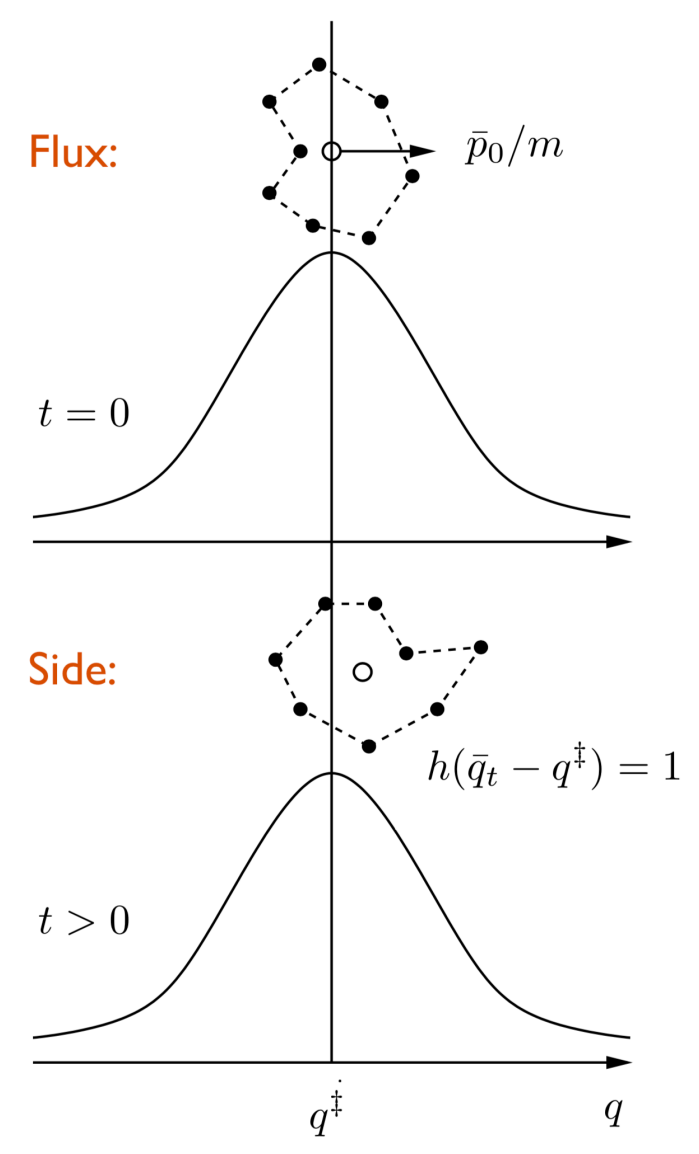

FIG. 3. Illustration of RPMD rate theory for a simple one-dimensional barrier transmission problem, with the dividing surface between reactants and products defined as in Eq. (20).

As we have already mentioned in the Introduction, it is straightforward to generalise RPMD rate theory to treat multi-dimensional reactions in their full dimensionality. The generalisation for condensed phase reactions was first described in Refs. 7 and 17, and an efficient implementation for gas phase bimolecular reactions was developed in Refs. 18 and 19. These generalisations have now been used in numerous calculations, ranging from studies of polyatomic reactions in the gas phase ${ }^{19} \sqrt{222}$ to reactions catalysed by enzymes ${ }^{23}$ and inorganic catalysts ${ }^{24}$ in solution. But how much confidence can we have in their results?

On the theoretical side, it was understood early on that RPMD rate theory becomes exact in the high temperature limit, and that it gives the exact quantum mechanical result for the shallow tunnelling through a parabolic barrier ${ }^{7}$ It is also parameter-free: a given reaction at a given temperature has a unique RPMD rate that is independent of the choice of dividing surface. And it is consistent with the quantum mechanical detailed balance condition: the 
$\mathrm{H}+\mathrm{H}_{2}$

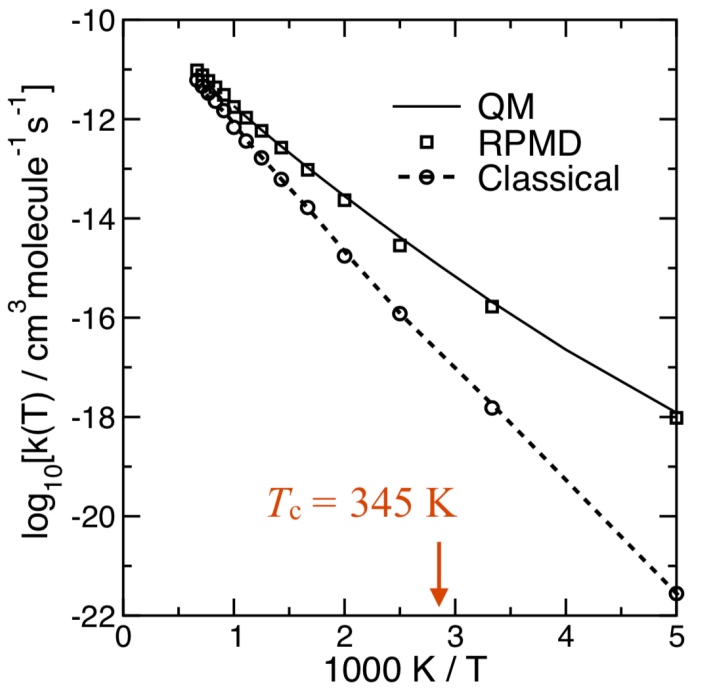

$\mathrm{F}+\mathrm{H}_{2}$

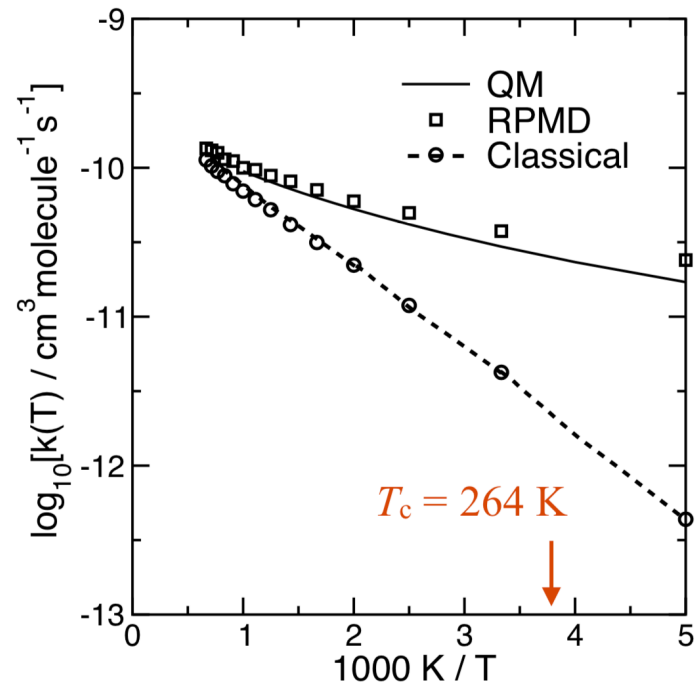

FIG. 4. Comparison of exact quantum mechanical (QM), RPMD, and classical rate constants for the gas phase $\mathrm{H}+\mathrm{H}_{2}$ and $\mathrm{F}+\mathrm{H}_{2}$ reactions. The critical temperature $T_{\mathrm{c}}$ in each panel marks the onset of the deep tunnelling instanton regime, as discussed in more detail in the text.

ratio of the forward and reverse RPMD rates is exactly equal to the quantum mechanical equilibrium constant for the reaction. These results are clearly encouraging, but they are not enough on their own to establish how reliable the RPMD approximation will be for any particular reaction at a given temperature.

For this, it is useful to consider some elementary gas phase reactions, for which the exact quantum mechanical rates can be computed for comparison by solving the Schrödinger equation. Two representative examples are shown in Fig. 4, which compares Arrhenius plots of the exact quantum mechanical, RPMD, and purely classical rate constants for the $\mathrm{H}+$ $\mathrm{H}_{2}$ and $\mathrm{F}+\mathrm{H}_{2}$ reactions. Here the exact rates were calculated using the ABC quantum reactive scattering program, 25 the RPMD rates using the method described in Ref. 18, and the classical rates using the same method with just $n=1$ ring polymer bead (see Ref. 18 for more details). One sees that the classical rates are almost perfectly Arrhenius for both reactions, whereas the quantum mechanical and RPMD rates exhibit significant 
non-Arrhenius curvature at low temperatures, as a result of tunnelling. At $200 \mathrm{~K}$, the exact quantum mechanical rate is more than three orders of magnitude larger than the classical rate for $\mathrm{H}+\mathrm{H}_{2}$, and more than an order of magnitude larger for $\mathrm{F}+\mathrm{H}_{2}$. But the RPMD rate is well within a factor of 2 of the exact quantum mechanical rate for both reactions: $k^{\mathrm{RPMD}}(T) / k^{\mathrm{QM}}(T)=0.79$ for $\mathrm{H}+\mathrm{H}_{2}$ and 1.46 for $\mathrm{F}+\mathrm{H}_{2}$ at $200 \mathrm{~K}$.

The critical temperatures labelled $T_{\mathrm{c}}$ in Fig. 4 mark the transitions from the shallow tunnelling regime to the deep tunnelling regime for each reaction. Above $T_{\mathrm{c}}$, the tunnelling is through the parabolic tip of the reaction barrier, and below $T_{\mathrm{c}}$ the parabolic approximation to the barrier breaks down and the reaction enters the "instanton" regime. The RPMD approximation is seen to overestimate the rate of the symmetric $\mathrm{H}+\mathrm{H}_{2} \rightarrow \mathrm{H}_{2}$ $+\mathrm{H}$ reaction in this regime, and to underestimate the rate of the asymmetric $\mathrm{F}+\mathrm{H}_{2} \rightarrow$ $\mathrm{HF}+\mathrm{H}$ reaction. This turns out to be a general feature: RPMD rate theory invariably overestimates the rates of symmetric reactions and underestimates the rates of asymmetric reactions at low temperatures. There are now many more examples of this, $\frac{18[19 \mid 26}{2}$ and it has been explained theoretically by Richardson and Althorpe ${ }^{27}$ from the connection between the optimum transition state theory approximation to the RPMD rate constant,

$$
k^{\mathrm{RPMD}, \mathrm{TST}}(T)=\frac{1}{Q_{r}(T)} \lim _{t \rightarrow 0_{+}} c_{f s}^{\mathrm{RPMD}}(t),
$$

and the rate constant given by the "Im F" version" ${ }^{28}$ of semiclassical instanton theory!

The transition to the instanton regime is illustrated schematically in Fig. 5, the left-hand side of which shows a ring polymer passing over a barrier. Consider first the parabolic approximation to the barrier around its maximum, $V(q) \simeq V\left(q^{\ddagger}\right)-m \omega_{b}^{2}\left(q-q^{\ddagger}\right)^{2} / 2$. Because this approximation is parabolic, and the springs that connect the beads of the ring polymer are harmonic, the overall potential experienced by the beads

$$
V_{n}(\mathbf{q}) \simeq \sum_{j=1}^{n}\left[\frac{1}{2} m \omega_{n}^{2}\left(q_{j}-q_{j+1}\right)^{2}+V\left(q^{\ddagger}\right)-\frac{1}{2} m \omega_{b}^{2}\left(q_{j}-q^{\ddagger}\right)^{2}\right]
$$

can be diagonalised with a normal mode transformation (which in this case can be written as a discrete Fourier transform or F.T.). The displacement of the centroid of the ring polymer from the barrier top, $\bar{q}-q^{\ddagger}$, is one of the normal modes, with imaginary frequency $\omega_{0}=i \omega_{b}$, and the lowest frequencies of the remaining (internal) modes of the ring polymer are $\omega_{ \pm k}=\sqrt{(2 \pi k / \beta \hbar)^{2}-\omega_{b}^{2}}$ for $k=1,2, \ldots$ There is thus a qualitative change in behaviour as $T$ passes through $T_{\mathrm{c}}=\hbar \omega_{b} / 2 \pi k_{\mathrm{B}}$. Above $T_{\mathrm{c}}$, all of the internal modes of the ring polymer 


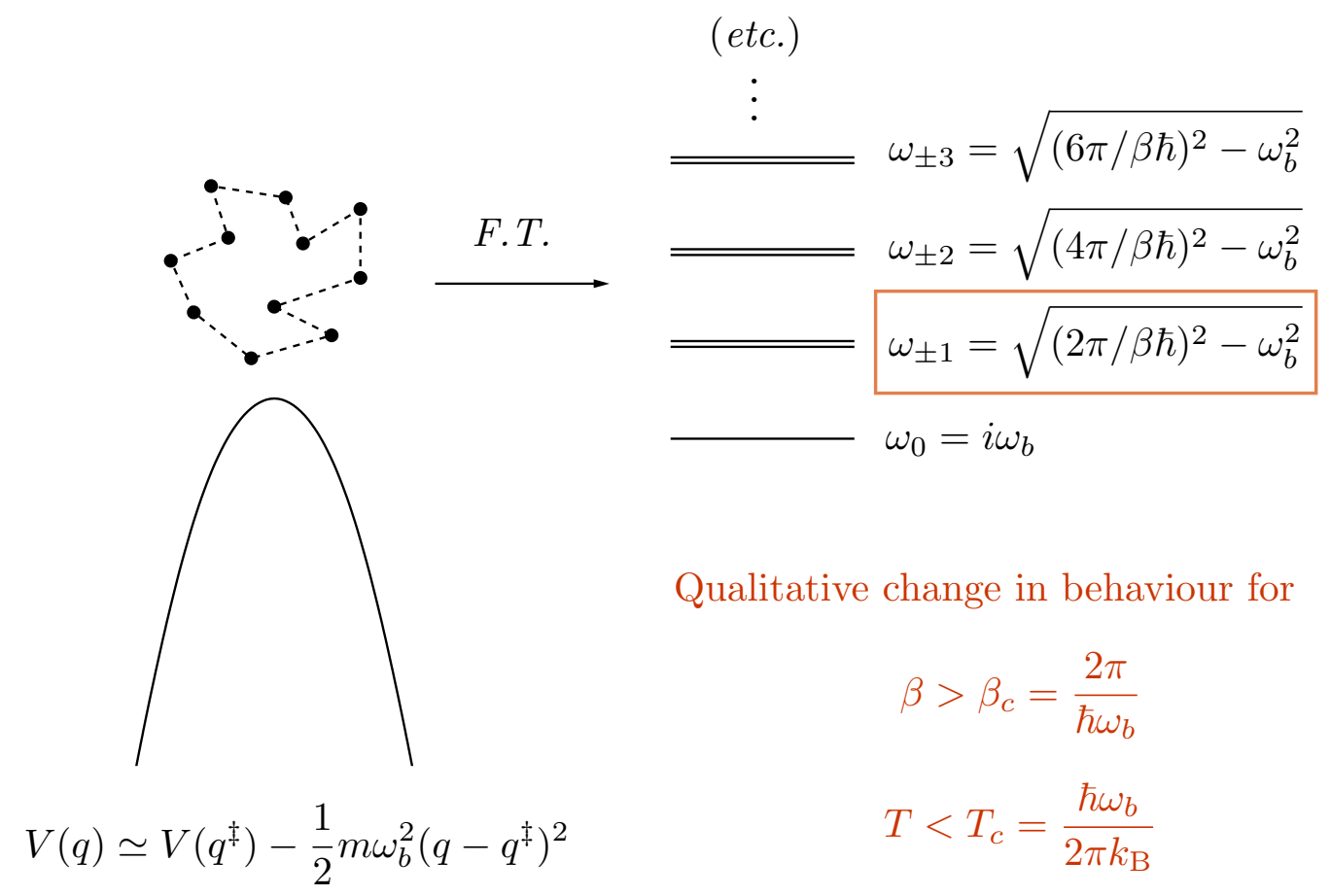

FIG. 5. Illustration of the transition from the shallow tunnelling regime to the instanton regime. See the text for a discussion.

have real frequencies, the configuration with each $q_{j}=q^{\ddagger}$ is a saddle point of the ring polymer potential energy surface $V_{n}(\mathbf{q})$, and the unstable mode that takes the system away from this saddle point is along the centroid coordinate $\bar{q}-q^{\ddagger}$ (which is therefore the correct "reaction coordinate" in this regime). But below $T_{\mathrm{c}}$, two or more of the internal modes of the ring polymer have imaginary frequencies at the configuration $q_{j}=q^{\ddagger}$. So this configuration is no longer a first order saddle point of $V_{n}(\mathbf{q})$, and one has to go beyond the parabolic approximation to the barrier to find one.

Richardson and Althorpe's key observation was that, for $T<T_{\mathrm{c}}$, the saddle point condition on the full ring polymer potential energy surface $V_{n}(\mathbf{q})$ (without making the parabolic barrier approximation)

$$
m \omega_{n}^{2}\left(q_{j+1}-2 q_{j}+q_{j-1}\right)=V^{\prime}\left(q_{j}\right)
$$

can be regarded (recalling that $\omega_{n}=1 / \beta_{n} \hbar$ ) as a finite difference approximation to Newton's 
second law

$$
m \frac{\mathrm{d}^{2} q}{\mathrm{~d}\left(\beta_{n} \hbar\right)^{2}}=V^{\prime}(q)
$$

for a purely classical trajectory on the "upside-down" barrier potential $-V(q)$. Furthermore, because of the cyclic ring polymer boundary condition $q_{n+1}=q_{1}$, this classical trajectory forms a periodic orbit, with period $n \times \beta_{n} \hbar=\beta \hbar$. There is therefore a direct connection between saddle points on the ring polymer potential energy surface and classical instanton trajectories. ${ }^{[29}$ And since these saddle points can be used to construct optimum ring polymer transition state dividing surfaces (through which the recrossing is expected to be minimised), there must also be a connection between RPMD-TST [Eq. (21)] and semiclassical instanton theory. 27

Richardson and Althorpe have explored this connection in detail, reaching a conclusion that is entirely consistent with the deep tunnelling $\left(T<T_{\mathrm{c}}\right)$ results in Fig. 4. They found that while the (optimum) RPMD-TST and "Im F" instanton rates both have the same exponential factor, involving the classical action around the instanton periodic orbit, they differ in the prefactor. Specifically, they found that the instanton rate coefficient, $k^{\text {inst }}(T)$, is related to a harmonic fluctuation approximation to the RPMD-TST rate coefficient, $k^{\mathrm{h}-\mathrm{RP}-\mathrm{TST}}(T)$, by 27

$$
k^{\text {inst }}(T)=\alpha_{\mathrm{h}}(T) k^{\mathrm{h}-\mathrm{RP}-\mathrm{TST}}(T)
$$

where $\alpha_{\mathrm{h}}(T)$ increases from 1 to 1.8 for a symmetric Eckart barrier and decreases from 1 to 0.6 for an asymmetric Eckart barrier as $T$ decreases from $T_{\mathrm{c}}$ to $T_{\mathrm{c}} / 2$. In our view this theoretical result, when combined with the results in Fig. 4 and similar results for other

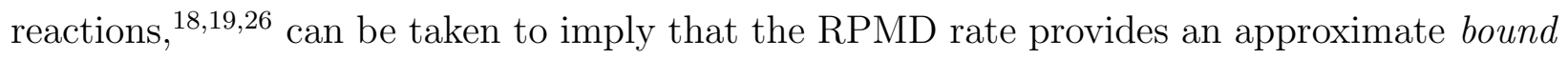
on the exact quantum mechanical rate in the deep tunnelling regime: an underestimate for symmetric reactions and an overestimate for asymmetric reactions which at reasonable temperatures is unlikely to be incorrect by a factor of more than 2 (in a regime where the classical rate is too small by several orders of magnitude).

This deals with tunnelling, but how well does the RPMD approximation capture the changes in zero point energy between the reactants and the transition state of a chemical reaction that are responsible for kinetic isotope effects? The strongest evidence we have for this is presented in Fig. 6, which compares Arrhenius plots of the exact quantum mechanical, $\mathrm{RPMD}$, and classical limit rate constants for the $\mathrm{Mu}+\mathrm{H}_{2}$ reaction. ${ }^{31}$ This reaction exhibits 

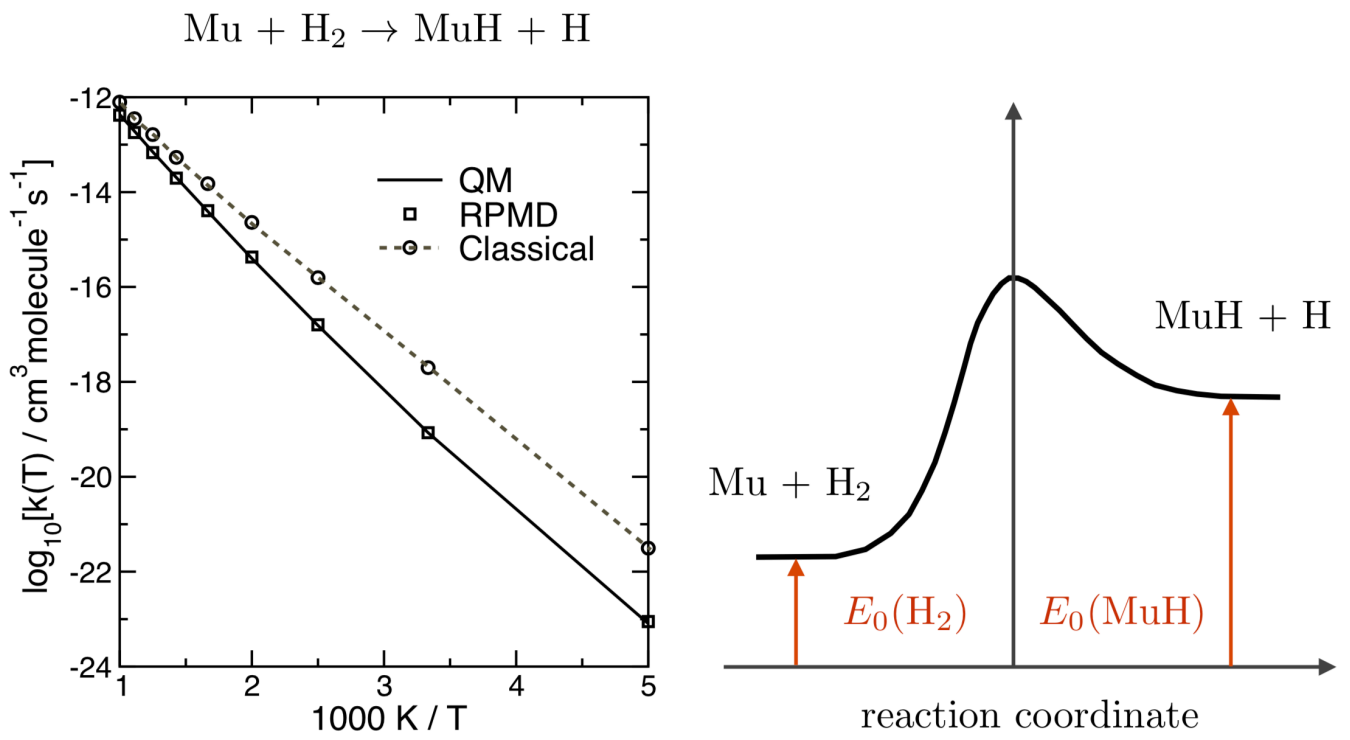

FIG. 6. Comparison of exact quantum mechanical, RPMD, and classical rate constants for the gas phase $\mathrm{Mu}+\mathrm{H}_{2} \rightarrow \mathrm{MuH}+\mathrm{H}$ reaction, which exhibits an extreme zero point energy effect (right).

an extreme change in zero point energy along the reaction coordinate as a result of the light mass of the muonium isotope, which has approximately one ninth the mass of a hydrogen atom. Because the zero point energy in the product $\mathrm{MuH}$ molecule (and also at the $\mathrm{Mu}-\mathrm{H}-\mathrm{H}$ transition state) is much larger than that in the $\mathrm{H}_{2}$ reactant molecule, the exact quantum mechanical rate constant is now significantly smaller than the classical limit rate constant, in contrast to the situation for $\mathrm{H}+\mathrm{H}_{2}$ in Fig. 4. And because the change in zero point energy along the reaction coordinate raises and broadens the reaction barrier, there is almost no perceptible tunnelling in the quantum mechanical calculation: the quantum and classical rate constants in Fig. 6 are both almost perfectly Arrhenius. The upshot of the figure is that the RPMD approximation captures the zero point energy effect extremely well: the difference between the RPMD and quantum rate constants is no more than $5 \%$ all the way down to $200 \mathrm{~K} ! 31$

We find this to be even more impressive than the way in which RPMD rate theory captures deep tunnelling. There have been many attempts over the years to "correct" purely classical trajectories to account for changes in zero point energy, ${ }_{32133}$ none of which has yet been 
found to be entirely satisfactory ${ }^{34}$ But for thermally averaged quantities such as thermal rate coefficients, the results in Fig. 6 seem to suggest that RPMD does this automatically. This has implications not just for gas phase reactions, but also for the absence of any "zero point energy leakage" issues in RPMD simulations of condensed phase systems such as liquid water. $\stackrel{35}{ }$

\section{WOLYNES THEORY}

So far we have only considered adiabatic chemical reactions which proceed on a single Born-Oppenheimer potential energy surface. Another important class of reactions are nonadiabatic reactions such as electron transfer and proton-coupled electron transfer reactions, in which there is a change in electronic state as the reaction proceeds.

Sticking with one-dimensional notation for simplicity, the Hamiltonian that governs these reactions has the form

$$
\hat{H}=\hat{H}_{0}|0\rangle\left\langle 0\left|+\hat{H}_{1}\right| 1\right\rangle\langle 1|+\Delta(|0\rangle\langle 1|+| 1\rangle\langle 0|)
$$

where $|0\rangle$ and $|1\rangle$ are the two electronic states and

$$
\hat{H}_{i}=\frac{\hat{p}^{2}}{2 m}+V_{i}(\hat{q})
$$

The last term in Eq. (26) is the diabatic coupling between the two electronic states, in which we have taken $\Delta$ to be a constant (the Condon approximation $\sqrt{36}$ ).

The exact quantum mechanical thermal rate constant for the transition from state $|0\rangle$ to state $|1\rangle$ can again be written in terms of a flux-side correlation function as in Eqs. (11) and (12), but now the reactant partition function is $Q_{r}(T)=\operatorname{tr}\left[e^{-\beta \hat{H}_{0}}\right]$, and the product side and reactive flux operators are $\hat{h}=|1\rangle\langle 1|$ and

$$
\hat{F}=\frac{i}{\hbar}[\hat{H}, \hat{h}]=\frac{i \Delta}{\hbar}(|0\rangle\langle 1|-| 1\rangle\langle 0|) .
$$

In fact, since $\hat{F}$ is the Heisenberg time derivative of $\hat{h}$, we can equally well replace Eq. (11) with

$$
k(T)=\frac{1}{2 Q_{r}(T)} \int_{-\infty}^{\infty} c_{f f}(t) \mathrm{d} t
$$

in which $c_{f f}(t)$ is a flux-flux correlation function: 16

$$
c_{f f}(t)=\operatorname{tr}\left[e^{-\beta \hat{H} / 2} \hat{F} e^{-\beta \hat{H} / 2} e^{+i \hat{H} t / \hbar} \hat{F} e^{-i \hat{H} t / \hbar}\right] .
$$


In the non-adiabatic limit where $\Delta \rightarrow 0$, it suffices to consider just the leading $O\left(\Delta^{2}\right)$ contribution to $k(T)$, which leads to the Fermi Golden Rule expression for the rate constant ${ }^{36}$

$$
k(T)=\frac{\Delta^{2}}{\hbar^{2} Q_{r}(T)} \int_{-\infty}^{\infty} \operatorname{tr}\left[e^{-\beta \hat{H}_{0}} e^{-i \hat{H}_{0} t / \hbar} e^{+i \hat{H}_{1} t / \hbar}\right] \mathrm{d} t .
$$

In a pioneering paper published over thirty years ago, Wolynes made a steepest descent approximation to the time integral in Eq. (31) to obtain ${ }^{9}$

$$
k(T) \simeq \frac{\Delta^{2}}{\hbar Q_{r}(T)} \sqrt{\frac{2 \pi}{-\beta F^{\prime \prime}\left(\lambda_{\mathrm{sp}}\right)}} e^{-\beta F\left(\lambda_{\mathrm{sp}}\right)},
$$

in which $F(\lambda)$ is an effective free energy defined by

$$
e^{-\beta F(\lambda)}=\operatorname{tr}\left[e^{-(\beta-\lambda) \hat{H}_{0}} e^{-\lambda \hat{H}_{1}}\right]
$$

and $\lambda_{\mathrm{sp}}$ can be found from the saddle point condition $F^{\prime}\left(\lambda_{\mathrm{sp}}\right)=0$. Noting that $e^{-\beta F(0)}=$ $\operatorname{tr}\left[e^{-\beta \hat{H}_{0}}\right]=Q_{r}(T)$, we see that Eq. (32) can be re-written as

$$
k(T) \simeq \frac{\Delta^{2}}{\hbar} \sqrt{\frac{2 \pi}{-\beta F^{\prime \prime}\left(\lambda_{\mathrm{sp}}\right)}} \exp \left[-\beta \int_{0}^{\lambda_{\mathrm{sp}}} F^{\prime}(\lambda) \mathrm{d} \lambda\right] .
$$

Since this only involves free energy derivatives, it is ideally suited to an imaginary time path integral (classical isomorphism) calculation.

An $n$-bead path integral discretisation of Eq. (33) can be constructed in the same way as the $n$-bead discretisation of $Q(T)=\operatorname{tr}\left[e^{-\beta \hat{H}}\right]$ in Eq. (3), the only difference being that one must now pay attention to which beads of the ring polymer necklace are on electronic state $|0\rangle$ and which on electronic state $|1\rangle$. This is straightforward to do, and when one does it one finds that the result has the same form as Eq. (3),

$$
e^{-\beta F\left(\lambda_{l}\right)}=\frac{1}{(2 \pi \hbar)^{n}} \int \mathrm{d}^{n} \mathbf{p} \int \mathrm{d}^{n} \mathbf{q} e^{-\beta_{n} H_{l}(\mathbf{p}, \mathbf{q})},
$$

in which $\lambda_{l}=l \beta_{n}$ for $l=0,1, \ldots, n$. Here

$$
\begin{aligned}
& H_{0}(\mathbf{p}, \mathbf{q})=h(\mathbf{p}, \mathbf{q})+\sum_{j=1}^{n} V_{0}\left(q_{j}\right), \\
& H_{n}(\mathbf{p}, \mathbf{q})=h(\mathbf{p}, \mathbf{q})+\sum_{j=1}^{n} V_{1}\left(q_{j}\right),
\end{aligned}
$$

and

$$
H_{l}(\mathbf{p}, \mathbf{q})=h(\mathbf{p}, \mathbf{q})+\frac{V_{0}\left(q_{1}\right)+V_{1}\left(q_{1}\right)}{2}+\sum_{j=2}^{l} V_{1}\left(q_{j}\right)+\frac{V_{0}\left(q_{l+1}\right)+V_{1}\left(q_{l+1}\right)}{2}+\sum_{j=l+2}^{n} V_{0}\left(q_{j}\right)
$$




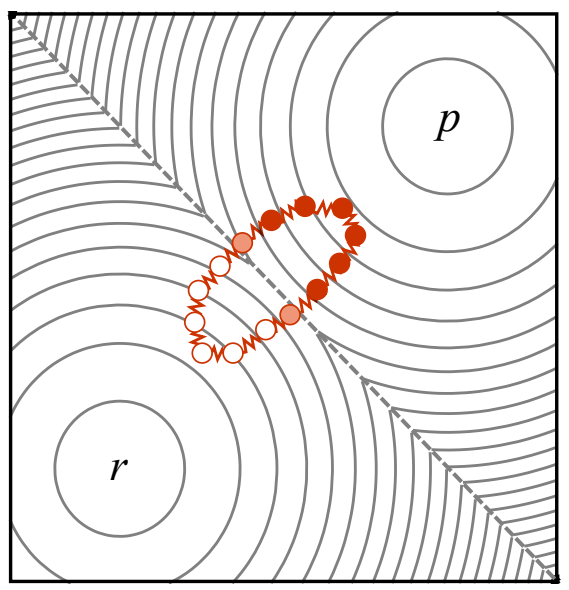

FIG. 7. Illustration of the path integral used in Wolynes theory when $l=n / 2$ and $\lambda_{l}=\beta / 2 . r$ and $p$ label the minima of the reactant and product electronic states of a non-adiabatic reaction. The hollow ring polymer beads are on the reactant surface, the filled beads on the product surface, and the shaded beads on the average of the two.

for $l=1, \ldots, n-1$, where $h(\mathbf{p}, \mathbf{q})$ is the free ring polymer Hamiltonian

$$
h(\mathbf{p}, \mathbf{q})=\sum_{j=1}^{n}\left[\frac{p_{j}^{2}}{2 m}+\frac{1}{2} m \omega_{n}^{2}\left(q_{j}-q_{j+1}\right)^{2}\right] .
$$

Using this discretisation, the derivatives of $F(\lambda)$ that are needed to evaluate Eq. (34) can be written as 37

$$
-\beta F^{\prime}\left(\lambda_{l}\right)=\left\langle s\left(q_{1}\right)\right\rangle_{\lambda_{l}}
$$

and

$$
-\beta F^{\prime \prime}\left(\lambda_{l}\right)=\left\langle s\left(q_{1}\right) s\left(q_{l+1}\right)\right\rangle_{\lambda_{l}}-\left\langle s\left(q_{1}\right)\right\rangle_{\lambda_{l}}^{2}
$$

where $s(q)=V_{0}(q)-V_{1}(q)$ and

$$
\langle\cdots\rangle_{\lambda_{l}}=\frac{\int \mathrm{d}^{n} \mathbf{p} \int \mathrm{d}^{n} \mathbf{q} e^{-\beta_{n} H_{l}(\mathbf{p}, \mathbf{q})}(\cdots)}{\int \mathrm{d}^{n} \mathbf{p} \int \mathrm{d}^{n} \mathbf{q} e^{-\beta_{n} H_{l}(\mathbf{p}, \mathbf{q})}}
$$

is a canonical average in the system with the ring polymer Hamiltonian $H_{l}(\mathbf{p}, \mathbf{q})$. For example, when $l=n / 2$ and $\lambda_{l}=\beta / 2$ this system has the ring polymer Hamiltonian illustrated in Fig. 7.

The ease with which Eqs. (40) and (41) can be evaluated makes Wolynes theory just as widely applicable to non-adiabatic reactions as RPMD rate theory is to adiabatic reactions. 
There is no problem in applying the theory to realistic (anharmonic and multi-dimensional) models of non-adiabatic reactions, and in fact this was possible even thirty years ago. For example Bader et al ${ }^{38}$ used Wolynes theory to investigate the role of nuclear tunnelling in a model of the aqueous ferrous-ferric electron transfer reaction in 1990, and Zheng et al. used it to study conformational reorganisation in a model of the electron transfer reactions of tuna cytochrome $\mathrm{c}$ in 1991. However, it seems to us that the theory has not had the recognition it deserves since then.

One possible reason for this is that there has been some confusion in the literature about the applicability of Wolynes theory to the Marcus inverted regime. ${ }^{40} F(\lambda)$ can only be computed from Eq. (33) when $\lambda$ lies in the range $0 \leq \lambda \leq \beta$, because the matrix elements of $e^{-x \hat{H}_{i}}$ become undefined for $x<0$. By the same token, $F^{\prime}(\lambda)$ and $F^{\prime \prime}(\lambda)$ can only be computed from the path integral averages in Eqs. (40) and (41) when $\lambda$ is in the same range. In the normal Marcus regime, this does not present any problem, because one can show that the saddle point value of $\lambda$ that enters the expression for the Wolynes theory rate constant in Eq. (34) does lie in this range: $\lambda_{\mathrm{sp}}$ moves from $\beta / 2$ for symmetric electron transfer $\left(\Delta_{r} \mathrm{G}^{\ominus}=0\right)$ to $\lambda_{\mathrm{sp}}=0$ for activationless electron transfer $\left(\Delta_{r} \mathrm{G}^{\ominus}=-\Lambda\right.$, where $\Lambda$ is the Marcus theory reorganisation energy). However, in the inverted regime, $\lambda_{\mathrm{sp}}$ becomes negative, and so inaccessible from Eqs. (33), (40) and (41), which has led to claims that Wolynes theory cannot be used in this regime. $\underline{40}$

These claims turn out to be incorrect, for the following reasons. One can certainly use Eqs. (33), (40) and (41) to calculate $F(\lambda)$ throughout the range $0 \leq \lambda \leq \beta$. Since the result is an analytic function of $\lambda$ within this range, it has a unique analytic continuation beyond the range, including a continuation to negative values of $\lambda$. This continuation can be constructed numerically by fitting $F(\lambda)$ to a suitable functional form, and when one does this one finds that the value of $\lambda_{\mathrm{sp}}$ in the inverted regime is typically very close to zero, so analytic continuation to the saddle point is numerically robust. ${ }^{37}$ Thus all of the quantities that are needed to evaluate Eq. (34) can reliably be calculated from accessible path integral simulations, and Wolynes theory can indeed be applied in the inverted regime.

As an example, Fig. 8 shows the results of such a calculation for a typical spin-boson model with parameters chosen to be representative of an electron transfer reaction in aqueous solution (a reorganisation energy of $50 \mathrm{kcal} / \mathrm{mol}$ from a Debye spectral density with a characteristic frequency of $500 \mathrm{~cm}^{-1}$, and a temperature of $\left.300 \mathrm{~K}\right) .37$ The figure compares 


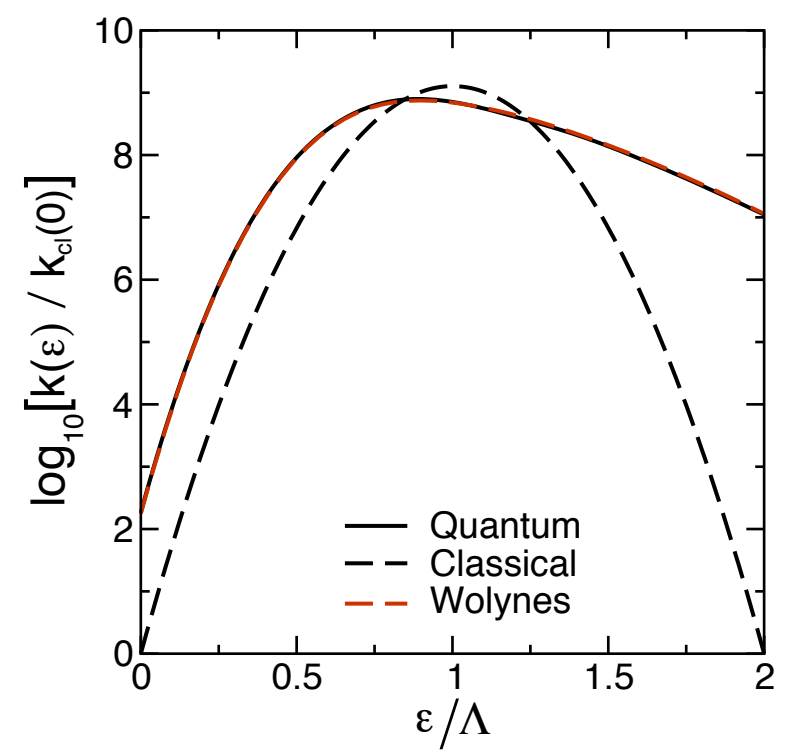

FIG. 8. Comparison of exact quantum mechanical, classical Marcus theory, and Wolynes theory rate constants for a typical spin-boson model, as a function of $\epsilon / \Lambda$.

the exact quantum mechanical, classical Marcus theory, and Wolynes theory rate constants as a function of $\epsilon / \Lambda$, where $\epsilon=-\Delta_{r} \mathrm{G}^{\ominus}$ is the thermodynamic driving force and $\Lambda$ is the reorganisation energy. $\epsilon / \Lambda=0$ corresponds to symmetric electron transfer in the normal regime, $\epsilon / \Lambda=1$ corresponds to activationless electron transfer, and $\epsilon / \Lambda=2$ is deep inside the Marcus inverted regime. One sees that tunnelling through the reaction barrier enhances the rate over the classical Marcus theory prediction by many orders of magnitude in the inverted regime, and that the Wolynes theory calculation performed by extrapolating imaginary time path integral data to $\lambda_{\mathrm{sp}}<0$ is just as accurate in this regime as it is in the normal regime. $\stackrel{37}{ }$ 
(a)

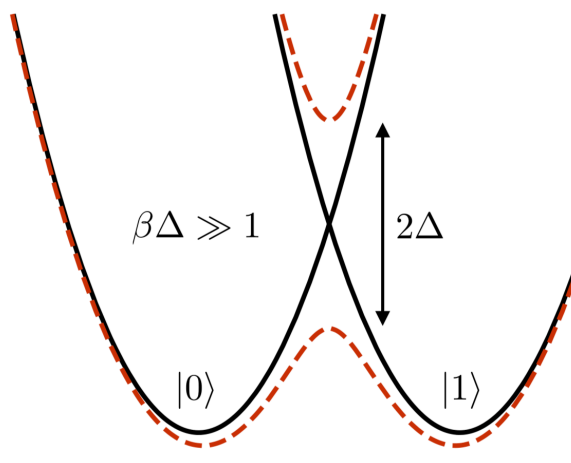

(c)
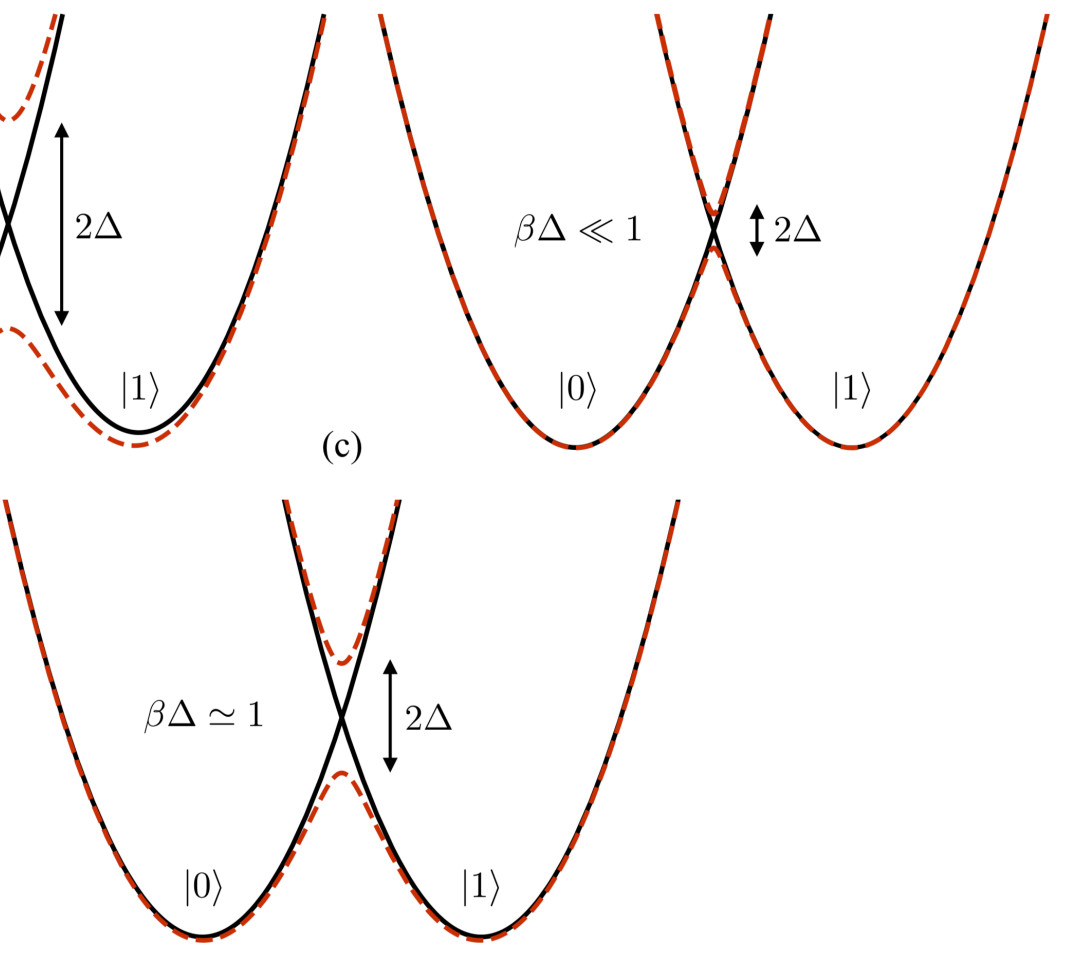

FIG. 9. Illustration of the adiabatic to non-adiabatic transition. The black curves are the electronic diabats and the red curves the electronic adiabats, both plotted as functions of the diabatic energy gap. (a) The adiabatic limit where $\beta \Delta \gg 1$ and the Born-Oppenheimer approximation is appropriate. (b) The non-adiabatic limit where $\beta \Delta \ll 1$ and Fermi's Golden Rule is appropriate. (c) The intermediate regime in which $\beta \Delta \simeq 1$ and neither is appropriate (on its own).

\section{ADIABATIC TO NON-ADIABATIC TRANSITION}

In the last two sections, we have established that RPMD rate theory provides a reliable way to include zero point energy and tunnelling effects in electronically adiabatic (BornOppenheimer) reaction rates, and that Wolynes theory provides a way to include these effects in electronically non-adiabatic (Fermi Golden Rule) rates. Both of these theories exploit the classical isomorphism, both are easily implemented, and both can routinely be applied to atomistic models of arbitrarily complex (multi-dimensional and anharmonic) systems.

However, this does not completely solve the chemical reaction rate problem in complex systems, because many reactions are not fully in either the adiabatic or the non-adiabatic 
limit. Fig. 9 illustrates the transition from adiabatic to non-adiabatic behaviour as the electronic coupling strength $\Delta$ decreases. In panel (a), $\beta \Delta \gg 1$, the excited adiabatic electronic state is thermally inaccessible from the ground state, and the Born-Oppenheimer rate on the ground adiabatic potential energy surface is all one has to compute. In panel (b), $\beta \Delta \ll 1$, and second order perturbation theory in $\Delta$ (Fermi's Golden Rule) suffices to give the correct reaction rate. But in panel (c), where $\beta \Delta \simeq 1$, neither the Born-Oppenheimer approximation nor Fermi's Golden Rule is reliable, so we need to find another way to compute the rate.

One thing that makes this difficult is the fact that, for condensed phase electron transfer reactions, it is not just the dimensionless electronic coupling strength $\beta \Delta$ that determines whether or not a reaction will be in the Golden-Rule regime. It is well known that solvent friction can drive a reaction away from this regime even when $\beta \Delta$ is relatively small. 14,45 There exist several simple theories which extend the classical Marcus theory of electron transfer away from the non-adiabatic limit. One of the best known of these, valid in the high temperature and high friction regime, is Zusman theory, ${ }^{41}$ which can be understood in terms of a Páde resummation of the leading terms in the perturbative series in $\Delta$. It is in principle possible to extend Wolynes theory beyond the non-adiabatic limit in the same way. However, in addition to requiring the fourth order contribution to the rate, which involves a triple integral in time, this sort of approach can only ever go some of the way towards the adiabatic limit. In the classical (high temperature) case, this difficulty has typically been overcome by connecting the Marcus theory rate to other known analytical results - such as Kramer's theory for the large $\Delta$ and high friction limit and the high friction cusped barrier result - with simple interpolation formulas. $\underline{46}+\underline{50}$

Inspired by these approaches, we have recently suggested a very simple interpolation formula that can be used to calculate the reaction rate at arbitrary values of the electronic coupling strength and which only requires the Golden Rule and Born-Oppenheimer rates as input: 10

$$
k(\Delta) \simeq \frac{k_{\mathrm{GR}}(\Delta) k_{\mathrm{BO}}(\Delta)}{k_{\mathrm{GR}}(\Delta)+k_{\mathrm{BO}}(\Delta=0)} .
$$

It is straightforward to show that this formula correctly approaches both the Golden Rule and Born-Oppenheimer limits, as illustrated in Fig. 10. As $\beta \Delta \rightarrow 0$ the denominator $k_{\mathrm{GR}}(\Delta)+k_{\mathrm{BO}}(\Delta=0)$ tends to $k_{\mathrm{BO}}(\Delta=0)$, which then cancels with the $k_{\mathrm{BO}}(\Delta)$ in the numerator to leave $k_{\mathrm{GR}}(\Delta)$, the correct non-adiabatic rate constant. And when $\beta \Delta \gg 1$, 


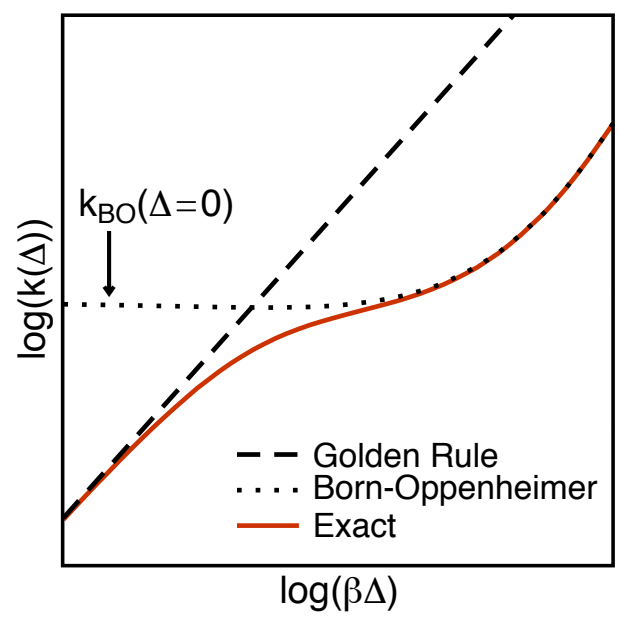

FIG. 10. Illustration of the transition from non-adiabatic (Fermi Golden Rule) to adiabatic (BornOppenheimer) behaviour of an electron transfer rate constant (red curve), as a function of $\beta \Delta$. Note the logarithmic scales on both axes.

$k_{\mathrm{GR}}(\Delta)+k_{\mathrm{BO}}(\Delta=0)$ tends to $k_{\mathrm{GR}}(\Delta)$, which then cancels with the same term in the numerator to leave $k_{\mathrm{BO}}(\Delta)$, the correct adiabatic rate constant. The interpolation formula can also be shown to obey detailed balance for any value of $\Delta$ : $k_{f}(\Delta) / k_{\mathrm{b}}(\Delta)=K(\Delta)$ where $k_{f}(\Delta)$ and $k_{b}(\Delta)$ are the (interpolated) forward and backward electron transfer rate constants and $K(\Delta)$ is the quantum mechanical equilibrium constant 10 And it reduces to the Zusman formula under the appropriate conditions (in the high friction and high temperature limit)! 10

Since Eq. (43) only requires the calculation of Golden Rule and Born-Oppenheimer rates, it can be combined with any methods that are able to calculate these rates. In particular, it can be combined with RPMD rate theory for the Born-Oppenheimer rate and Wolynes theory for the Golden Rule rate, and so used to include nuclear quantum effects in simulations of condensed phase electron transfer reactions with arbitrary electronic coupling strengths (at least in the normal Marcus regime where it is possible to define a configuration space dividing surface between the reactants and products and use this in RPMD rate theory to 
calculate the Born-Oppenheimer rate).

We have recently tested the accuracy of Eq. (43) by comparison with exact quantum mechanical results for a variety of problems, including a simple one-dimensional non-adiabatic scattering problem and a series of highly demanding spin-boson models (with different thermodynamic driving forces, reorganisation energies, solvent frictions, reaction coordinate frequencies, and electronic coupling strengths) ${ }^{10}$ The formula was found to work remarkably well in all regimes considered, giving results within a factor of 2 of the exact quantum mechanical results even in the most deep tunnelling of cases. Since this is comparable to the accuracy of RPMD rate theory in the adiabatic limit, and of Wolynes theory in the nonadiabatic limit, it is unlikely that one could do any better in trying to extend these theories to treat general electronically non-adiabatic reactions. And the interpolation formula really is remarkably simple to implement, even for realistic (anharmonic and multi-dimensional) models of chemical reactions, because it simply involves the combination of these two wellestablished and thoroughly tested techniques.

\section{SUMMARY}

In this review, we have argued that methods based on the classical isomorphism can be used to calculate accurate quantum mechanical reaction rates in a wide variety of regimes, from fully adiabatic to fully non-adiabatic and just about everywhere in between (the only exception being rates away from the Golden Rule limit in the Marcus inverted regime). Quantum mechanical effects can be quite enormous in chemical reactions, even at room temperature, so it is important to have methods that are able to predict these effects. The classical isomorphism is ideal in this respect, because it provides a very natural way to capture the dominant quantum effects in reaction rates (tunnelling - which gives rise to non-Arrhenius behaviour at low temperatures, and zero-point energy - which is responsible for both normal and inverse kinetic isotope effects). There are many other problems that the isomorphism can be used for, several of which (such as the vibrational spectroscopy of liquids and biomolecules) are discussed in other contributions to this Discussion. But the chemical reaction rate problem is undoubtedly its "killer application". 
1 See, e.g., K. J. Laidler, Chemical Kinetics, 3rd Edition, Harper Collins, New York, 1987.

2 K. Liu, R. T. Skodje and D. E. Manolopoulos, Phys. Chem. Comm. 2002, 5, 27.

3 J. B. Kim, M. L. Weichmann, T. F. Sjolander, D. M. Neumark, J. Klos, M. H. Alexander and D. E. Manolopoulos, Science 2015, 349, 510.

4 D. Chandler and P. G. Wolynes, J. Chem. Phys. 1981, 74, 4078.

5 M. Parrinello and A. Rahman, J. Chem. Phys. 1984, 80, 860.

6 I. R. Craig and D. E. Manolopoulos, J. Chem. Phys. 2004, 121, 3368.

7 I. R. Craig and D. E. Manolopoulos, J. Chem. Phys. 2005, 122, 084106.

8 I. R. Craig and D. E. Manolopoulos, J. Chem. Phys. 2005, 123, 034102.

9 P. G. Wolynes, J. Chem. Phys. 1987, 87, 6559.

10 J. E. Lawrence, T. Fletcher, L. P. Lindoy and D. E. Manolopoulos, J. Chem. Phys. 2019, 151, 114119.

11 S. Habershon, D. E. Manolopoulos, T. E. Markland and T. F. Miller III, Annu. Rev. Phys. Chem. 2013, 64, 387.

12 T. F. Miller III and D. E. Manolopoulos, J. Chem. Phys. 2005, 122, 184503.

13 T. E. Markland, S. Habershon and D. E. Manolopoulos, J. Chem. Phys. 2008, 128, 194506.

14 S. Habershon, T. E. Markland and D. E. Manolopoulos, J. Chem. Phys. 2009, 131, 024501.

15 J. R. Cendagorta, A. Powers, T. J. H. Hele, O. Marsalek, Z. Bacic and M. E. Tuckerman, Phys. Chem. Chem. Phys. 2016, 18, 32169.

16 W. H. Miller, S. D. Schwartz and J. W. Tromp, J. Chem. Phys. 1983, 79, 4889.

17 R. Collepardo-Guevara, I. R. Craig and D. E. Manolopoulos, J. Chem. Phys. 2008, 128, 144502.

18 R. Collepardo-Guevara, Y. V. Suleimanov and D. E. Manolopoulos, J. Chem. Phys. 2009, 130 174713.

19 Y. V. Suleimanov, R. Collepardo-Guevara and D. E. Manolopoulos, J. Chem. Phys. 2011, 134, 044131.

20 Y. Li, Y. V. Suleimanov, M. Yang, W. H. Green and H. Guo, J. Phys. Chem. Lett. 2012, 4, 48.

21 Y. Li, Y. V. Suleimanov, J. Li, W. H. Green and H. Guo, J. Chem. Phys. 2013, 138, 094307.

22 J. W. Allen, W. H. Green, Y. Li, H. Guo and Y. V. Suleimanov, J. Chem. Phys. 2013, 138, 221103. 
23 N. Boekelheide, R. Salomon-Ferrer and T. F. Miller III, Proc. Nat. Acad. Sci. USA 2011, 108, 16159.

24 J. S. Kretchmer and T. F. Miller III, Inorg. Chem. 2016, 55, 1022.

25 D. Skouteris, J. F. Castillo and D. E. Manolopoulos, Comp. Phys. Comm. 2000, 133, 128.

26 Y. V. Suleimanov, R. Perez de Tuleda, P. G. Jambrina, J. F. Castillo, V. Saez-Rabanos, D. E. Manolopoulos and F. J. Aoiz, Phys. Chem. Chem. Phys. 2013, 15, 3655.

27 J. O. Richardson and S. C. Althorpe, J. Chem. Phys. 2009, 131, 214106.

28 C. G. Callan and S. Coleman, Phys. Rev. D 1977, 16, 1762.

29 W. H. Miller, J. Chem. Phys. 1975, 62, 1899.

30 S. C. Althorpe, J. Chem. Phys. 2011, 134, 114104.

31 R. Perez de Tudela, F. J. Aoiz, Y. V. Suleimanov and D. E. Manolopoulos, J. Phys. Chem. Lett. 2012, 3, 493.

32 J. M. Bowman, B. Gazdy and Q. Sun, J. Chem. Phys. 1989, 91, 2859.

33 W. H. Miller, W. L. Hase and C. L. Darling, J. Chem. Phys. 1989, 91, 2863.

34 T. D. Sewell, D. L. Thompson, J. D. Gezelter and W. H. Miller, Chem. Phys. Lett. 1992, 193, 512.

35 S. Habershon and D. E. Manolopoulos, J. Chem. Phys. 2009, 131, 244518.

36 A. Nitzan, Chemical Dynamics in Condensed Phases, Oxford University Press, Oxford, 2006.

37 J. E. Lawrence and D. E. Manolopoulos, J. Chem. Phys. 2018, 148, 102313.

38 J. S. Bader, R. A. Kuharski and D. Chandler, J. Chem. Phys. 1990, 93, 230 (1990).

39 C. Zheng, J. A. McCammon and P. G. Wolynes, Chem. Phys. 1991, 158, 261.

40 J. O. Richardson and M. Thoss, J. Chem. Phys. 2014, 141, 074106.

41 L. D. Zusman, Chem. Phys. 1980, 49, 295.

42 A. Garg, J. N. Onuchic and V. J. Ambegaokar, J. Chem. Phys. 1985, 83, 4491.

43 J. T. Hynes, J. Phys. Chem. 1986, 90, 3701.

44 I. Rips and J. Jortner, J. Chem. Phys. 1987 87, 2090.

45 M. Sparpaglione and S. Mukamel, J. Chem. Phys. 1988, 88, 3263.

46 D. F. Calef and P. G. Wolynes, J. Phys. Chem. 1983, 87, 3387.

47 E. Pollak, J. Chem. Phys. 1990, 93, 1116.

48 I. Rips and E. Pollak, J. Chem. Phys. 1995, 103, 7912.

49 A. Starobinets, I. Rips and E. Pollak, J. Chem. Phys. 1996, 104, 6547. 
50 V. Gladkikh, A. I. Burshtein and I. Rips, J. Phys. Chem. A 2005, 109, 4983. 